\title{
Simulation Study on Blood Flow Mechanism of Vein in Existence of Different Thrombus Size
}

\author{
Nabilah Ibrahim ${ }^{1}$ \\ Faculty of Electrical and Electronic Engineering \\ University Tun Hussein Onn Malaysia \\ 86400 Batu Pahat, Johor, Malaysia
}

\author{
Nur Shazilah Aziz ${ }^{2}$ \\ Department of Test \\ Venture Technocom Systems Sdn. Bhd. \\ Tebrau, 81100, Johor, Malaysia
}

\author{
Muhammad Kamil Abdullah ${ }^{3}$ \\ Faculty of Mechanical and Mechatronics \\ University Tun Hussein Onn Malaysia \\ 86400 Batu Pahat, Johor, Malaysia
}

\author{
Gan Hong Seng ${ }^{4}$ \\ Medical Engineering Technology \\ University Kuala Lumpur British Malaysia Institute \\ 53100, Gombak, Selangor, Malaysia
}

\begin{abstract}
Blood velocity is expected to be as a parameter for detecting abnormality of blood such as the existence of thrombus. Proper blood flow in veins is important to ensure effective return of deoxygenated blood to the heart. However, it is much challenging to recognize the vessel condition due to the inability to visualize the thrombus presence in the vessel. The presence of noise in the image obtained from ultrasound scanning is one of the obstructions in recognizing it. Considering the difficulty, this study aims to assess the velocity and vorticity at the vein valve region using Computational Fluid Dynamics (CFD) method. The velocity of blood and the size of valve orifice are considered important parameters in designing the vein since the stenosis and irregularities of velocity in blood vessels are known as the risk factors for thrombus formation. From the simulation, the velocity contour plot of the blood flow can be visualized clearly. The blood distribution was presented using velocity profile while the fluid particles movement was shown by the velocity vector. The low blood velocity clearly shows the low velocity region which reside at the cusps area and at the beginning of the valve leaflets. Therefore, the present study is able to visualize and evaluate the probable location of thrombus development in the blood vessel.
\end{abstract}

Keywords-Blood velocity profile; velocity contour plot; computational fluid dynamic (CFD); thrombus; vein valve

\section{INTRODUCTION}

Deep vein thrombosis (DVT) and pulmonary embolism (PE) that relatively known as venous thromboembolism is the third leading cause of cardiovascular disorder after myocardial infarction and stroke. DVT is the development or existence of blood clot or thrombus in one of the large veins deep in the body. The lower limb is the most common site of thrombosis due to relatively slow or disturbed blood flow. The existence of thrombus may block the flow of blood through the vein partially or completely. This may lead to the rupture of the thrombus which then migrates to lungs that finally becomes embolus to occlude a pulmonary artery. This condition is called PE. According to Virchow triad theory [1], at least two of the factors occurring simultaneously increase patient risk of developing DVT. The factors are stasis, vessel damage and hypercoagulability. Stasis is believed to be one of predominant of the three factors. In addition to stasis, endothelial damage also affecting the blood flow in disruption of vessel elasticity [2]. Dysfunction of endothelial increases the expression of adhesion molecules such monocytes, leukocytes, and platelets that later contribute to the abnormalities in blood flows. This is the primary factor to lead to hypercoagulability [3]. A lower extremity DVT linked to cause an estimated 50\% risk of PE if not treated in a timely effective manner. It is reported that $15 \%$ to $32 \%$ of lower extremity DVT most likely to develop PE [4]. Therefore, early diagnosis of DVT is essential to prevent unnecessary deaths from PE.

Although many diagnostic tools exist to evaluate the presence of DVT such as computed tomography (CT) and magnetic resonance imaging (MRI), the use of ultrasound (US) imaging for routine DVT evaluation is superior in accuracy, cost and feasibility [5]. On top, compression ultrasound and duplex ultrasonography are both available US methods to evaluate DVT by assessing the collapsibility of vein that may rapidly performed. Other than that, the evaluation of blood flow through a vessel is an essential aspects of cardiovascular health since it is known as primary factor that contribute to the death [6]. However, such methods are not always indicating the true behavior of blood flow especially for the complex geometry and vortex formation. Normally, the blood flow can be monitored non-invasively using ultrasound Doppler or by building a phantom mimicking the artery or vein for in-vitro test [7]. Performing such experiments usually was believed to giving out accurate result if properly executed following the biological nature. However, due to the difficulty in setting up the components, several results may come to error. Thus, the outcome might not be accurately reflecting the actual flowing of blood behavior.

An alternative to constructing a physical experiment is by performing a simulation of an actual conditions for virtually problem conditions. Computational fluid dynamics (CFD) investigation have been utilized to assess particular parameters in fluid flow, for instance wall shear stress, velocity of blood flow, and pressure [8]. Thus, CFD modelling is proposed to 
better understand the mechanism of underlying DVT. Previously, we have reported in [9] the CFD simulation on blood velocity and vorticity in vessel. However, the effect on the thrombus existence did not be considered. An efficient visualization of flow field can assist in further diagnosing the cause of vein disease. This study focus to investigate the effect of valve opening, and velocity to the thrombus formation on blood flow distribution in popliteal vein.

Next section shows some previous works conducted by researchers that related to computational simulation. In Section III, vein modelling is described which using computational domain. Simulation results are discussed in Section IV. Further discussion is elaborated in Section V. Finally, the conclusions and future work are presented in Section VI.

\section{PREVIOUS WORKS}

In medical education, prediction is much more important in the way to prepare for the necessary technique and aggressive motion on patients. In particular interest for relatively non-invasive technique and low cost, ultrasound techniques are mostly favorable to be used [10-11]. Those techniques may provide results on early analysis of certain disease. Nonetheless, the internal organs are unpredictable which may lead to less reproducibility results. Here, computational simulations or numerical studies for blood flow have always preferred as it is always a realistic way to simulate organ or vessel. Some studies show the findings from the simulation works. In [12], they make comparison between non-Newtonian and Newtownian models behavior of the blood to the plaque. In the study, the pulsatile flow was used interaction with lipid pool was observed. From the simulations, it is found that the non-Newtonian model shows a higher peak for most critical parameter considering the risk or benefit ratio for carotid endarectomy. The result was then increase understanding on the plaque stability. Oppositely, the study expressed the features limitation when considering only the wall shear stress and vomises stress. These parameters even though give benefit to predict the clot formation, it is insufficient to announce the flow distribution changes along the blood vessel. Therefore, the blood flow velocity, volume flow rate and shear distribution were analyzed in [13]. The work however becomes more complex when it dealing with angio-Computed Tomography data. Moreover, the study did not mention the analysis effect of blood flow velocity and vorticity on the plaque that might be considered as one of the factor that could trigger the plaque rupture. Thus, in [14], the work discussed on the possible plaque and thrombus rupture due to the interaction with blood flow. Even though the discussion proposed in detail the numerical work on blood flow velocity which also covered monocytes motion, it did not confer with the velocity vector for each minor segment along the vessel including the plaque and thrombus region. The information of velocity profile and velocity vector is essential to predict the probable location of thrombus formation in blood vessel.

\section{VEIN MODELLING}

After completing the vein drawing, the drawing will be used into another computer simulation program known as computational fluid dynamic (CFD) Ansys-CFX. Ansys-CFX is a type of software where users are allowing to test a system by simulating the fluid behavior in virtual environment. In this software, the simulation was divided into three cases. Sizes of valve aperture, velocity and sizes of thrombus will be the variable in the simulation. This is to ensure each changes in vein is fully discovered from the simulation.

\section{A. Computational Domain}

Computational domain is a platform of solving the mathematical model of the physical problem. Throughout the mathematical works, conservation of matter, momentum, and energy must be satisfied in the region of interest. Discretization technique was applied to develop approximations of the governing equations of fluid mechanics in the fluid region of interest. This discretized domain is known as grid or mesh. In this study, there is only one computational domain that will be focusing on which is venous vessel, as shown in Fig. 1.

Fig. 1 shows the structure of vessel with dimension which been drawing using SolidWorks. Fig. 1(a) indicates the length of vein, diameter, and diameter at swell area which are $10 \mathrm{~cm}$, $10 \mathrm{~mm}$, and $11 \mathrm{~mm}$ respectively. Noted that the blue arrow denotes the direction of blood flow. The length of the valve was assumed to $5 \mathrm{~mm}$ and the valve leaflets thickness assumed to be $1 \mathrm{~mm}$. Fig. 1(b) shows the vein model with the presence of thrombus. The thrombus size used in this study is $1 \mathrm{~mm}$ and $3 \mathrm{~mm}$ where the ration of $t: l$ is $1: 4$.

Fig. 2 shows the computational domain with the implemented mesh. Here, the mesh used is tetrahedral mesh where the mesh containing structured and unstructured mesh. The body is accommodated by unstructured mesh while, for the near wall region, structured mesh or known as inflation is used. The structured mesh is implemented due to the needs of critical meshing at the near wall region.

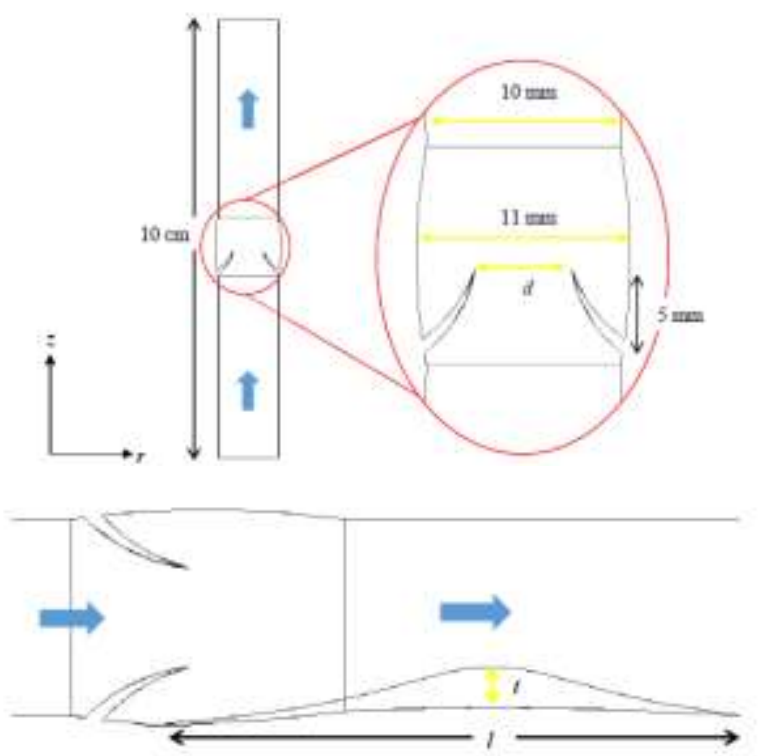

Fig. 1. Geometrical Structure of the Popliteal Vein with Dimension, a) Condition without Thrombus with $d$ Represent the Size of Leaflets Opening that vary from $30 \%$ to $70 \%$, b) DVT Condition with $t$ Represent Thrombus Size and $l$ Represent the Length of Thrombus. 


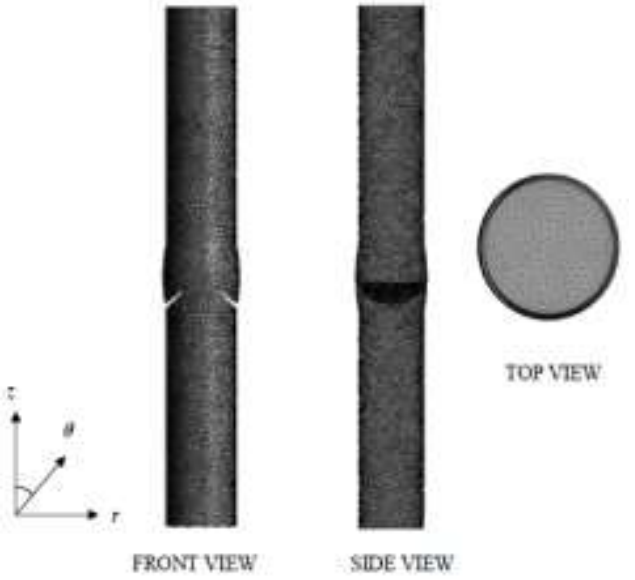

Fig. 2. Computational Domain Meshes for Front, Size and Top Views.

\section{B. Boundary Condition and Numerical Setup}

The boundary condition is to set the initial value of simulation. Here, the inlet, outlet and wall need to be assigned. Inlets are used for regions where inflow is expected while outlets are used for regions where outflow is expected.

Fig. 3 shows the specific location of the boundary applied on computational domain. The arrow denotes the direction of blood flow. Table I shows the details of boundary conditions.

The present numerical investigation was carried out with the employment of laminar model. All the numerical and boundary conditions was referred from the previous study in order to follow the real physiologic condition. Table II shows the details of boundary conditions.

\section{Code of Cases}

Fig. 4 shows the code uses for each type of cases. V10 represents the value of inlet velocity used. In this study, the range of velocity used was $10-50 \mathrm{~cm} / \mathrm{s}$. This is following the normal range of flow velocity in vein which actually from 10 $\mathrm{cm} / \mathrm{s}$ to $40 \mathrm{~cm} / \mathrm{s}$ [15]. While $20 \mathrm{~cm} / \mathrm{s}$ was chosen to simulate the cases with DVT condition as to represent the normal velocity flow in resting condition [16]. O3 indicates the size of valve orifice. While NT represents case without the presence of thrombus, $\mathrm{T} 1$ and $\mathrm{T} 3$ represent the case with the presence of $1 \mathrm{~mm}$ and $3 \mathrm{~mm}$ thrombus, respectively. $1 \mathrm{~mm}$ size of thrombus was chosen to represent the early formation of thrombus. Meanwhile, $3 \mathrm{~mm}$ thrombus was to represent the severe state of DVT condition.

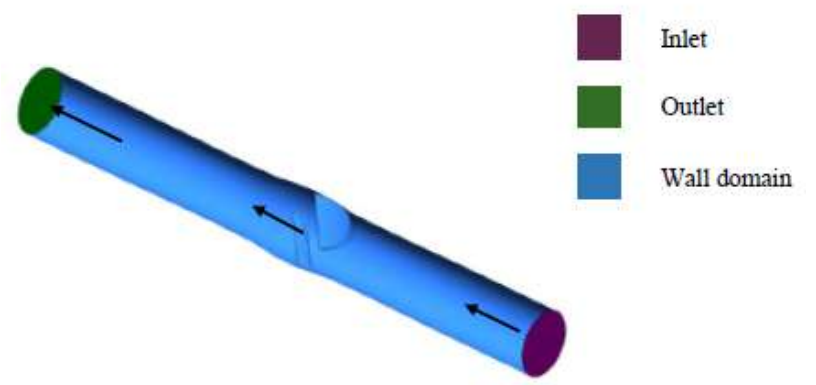

Fig. 3. Boundary Condition Location.
TABLE I. BOUNDARY CONDITION DETAILS AND FLOW PROPERTIES

\begin{tabular}{|l|l|}
\hline Blood Viscosity $(\mu)$ & 0.0035 Pa s $[16,19-20]$ \\
\hline Blood Density $(\rho)$ & $1050 \mathrm{kgm}^{-3}[21-24]$ \\
\hline Temperature & $37[17,18]$ \\
\hline Inlet $(\mu)$ & $10,20,30,40,50 \mathrm{cms}^{-1}[16]$ \\
\hline Outlet $(P)$ & $0 \mathrm{~mm} \mathrm{Hg}$ \\
\hline Wall & No-slip wall condition \\
\hline
\end{tabular}

TABLE II. NUMERICAL SETUP

\begin{tabular}{|l|l|}
\hline State of fluid flow & Steady \\
\hline & Residual type = RMS \\
Convergence criteria & Residual target =1.E- 4 \\
& Minimum iterations $=1$ \\
& Maximum iterations = 100 \\
\hline Model type & Laminar \\
\hline
\end{tabular}

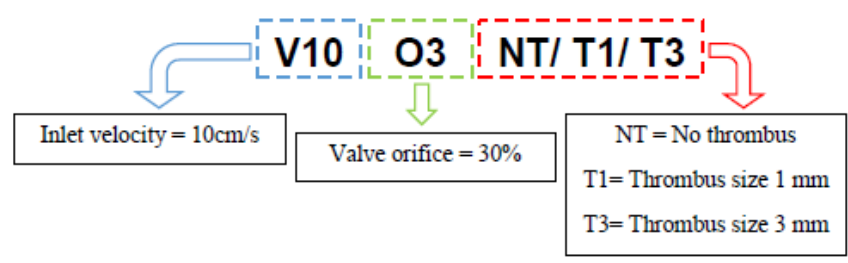

Fig. 4. Code uses for each Type of Cases.

\section{RESULTS}

The results will be including the velocity contour, velocity profile, velocity vector and also vorticity of the blood flow in the vein. The variable that changes in the simulation is the size of valve orifice, velocity and thrombus size. The valve aperture was set from $3 \mathrm{~mm}$ to $7 \mathrm{~mm}$, while velocity was set from $10 \mathrm{~cm} / \mathrm{s}$ to $50 \mathrm{~cm} / \mathrm{s}$. Thrombus size used was $1 \mathrm{~mm}$ and 3 $\mathrm{mm}$. This is to observe the blood behavior as it passes through stenosis or blockage in the blood stream.

\section{A. Effect of Different Velocity to the Blood Flow}

The information regarding blood velocity in a blood vessel might be an estimate of sufficient accuracy in many cases. Thus, this study was carried out to show the effect of blood velocity on the blood behavior at the valve region. This section was simulated to find the effect of different inlet velocity to the fluid flow inside the vessel. The different inlet velocity is representing the different velocity of human as every human carries different value of velocity insider their vessel.

Based on Fig. 5, the scaled legend of red color represents natural direction of blood flow which has the range of velocity from $5 \mathrm{~cm} / \mathrm{s}$ until $95 \mathrm{~cm} / \mathrm{s}$. While blue color represents the opposite direction of blood flow which the range of velocity between $5 \mathrm{~cm} / \mathrm{s}$ and $-5 \mathrm{~cm} / \mathrm{s}$. It is clearly can be visualized the increasing of velocity when the fluid is passing through the valve region and keep extended to the distal side, especially for the inlet velocity from $20 \mathrm{~cm} / \mathrm{s}$ until $50 \mathrm{~cm} / \mathrm{s}$. While low blood velocity region can be spotted reside in the valve cusps. The low velocity causing stagnation of blood where some particles of blood will be circling and pooling at the same place that could increase the blood contact time with the endothelial, thus will lead to formation of thrombus. 


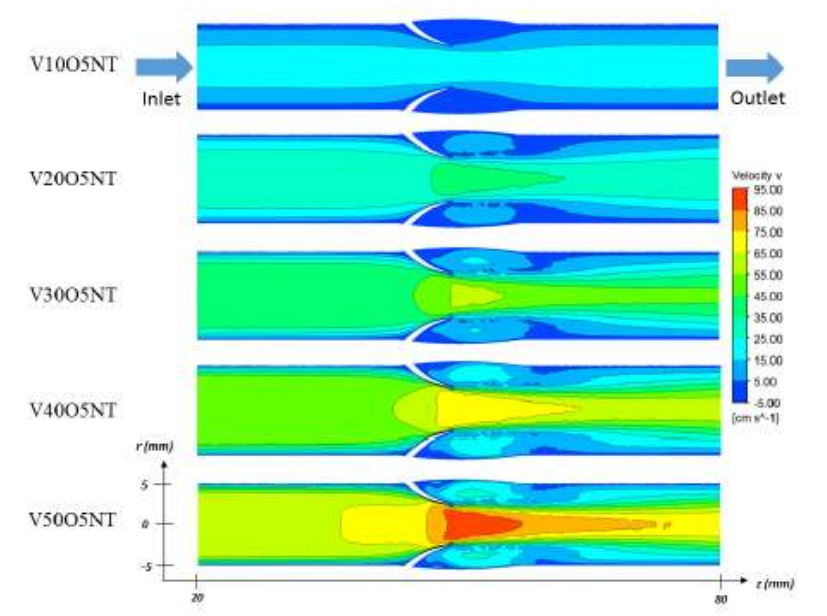

Fig. 5. The Various Value of Inlet Velocity with Valve Orifice Size $5 \mathrm{~mm}$.

Fig. 6 demonstrates velocity profile for five cases with different inlet velocity. The velocity profile was divided into five section along the vessel. Two sections which A and B was plotted prior the valve while section $\mathrm{C}, \mathrm{D}$, and $\mathrm{E}$ was plotted after the valve. Each sections were set at $8 \mathrm{~mm}$ interval. In all cases, velocity profile in section A and B shows fully developed with a slight steeper increased proportionally to the inlet velocity. At the section C of V20O5NT, the velocity value started to have negative value at the vessel wall. The reverse flow can be spotted reside at the vessel wall. This condition continues until the inlet velocity reach $50 \mathrm{~cm} / \mathrm{s}$. As the velocity reach further away from the wall at section D and $\mathrm{E}$, the fluid velocity changes from zero at the surface because of the no-slip condition.

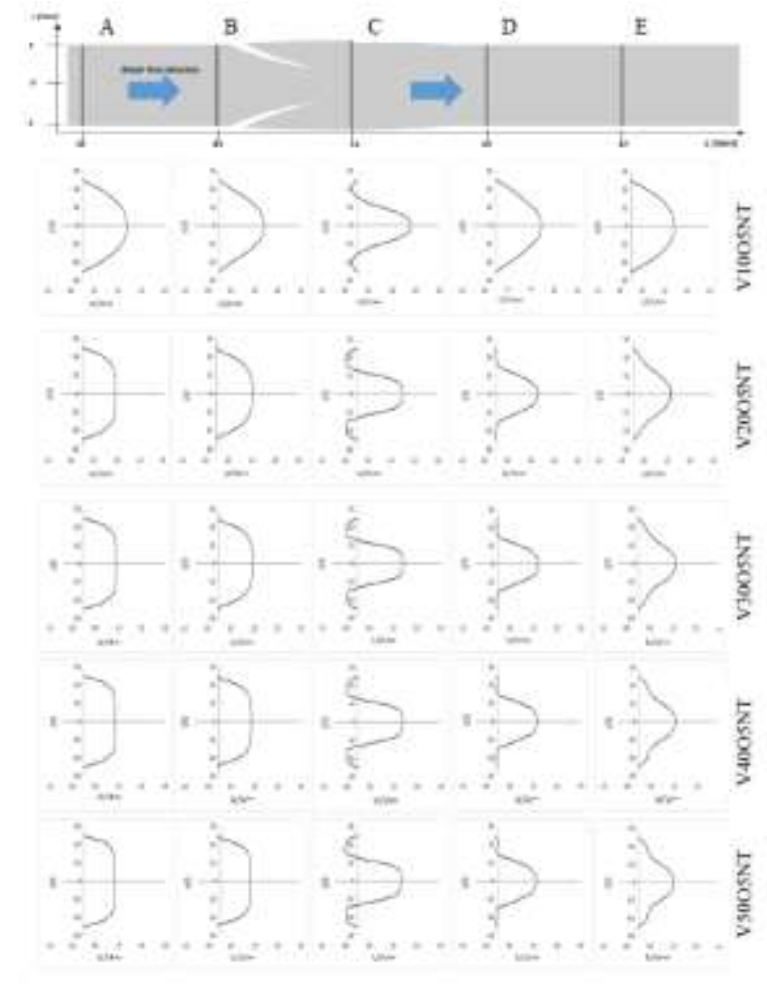

Fig. 6. Velocity Profile for different Inlet Velocity.

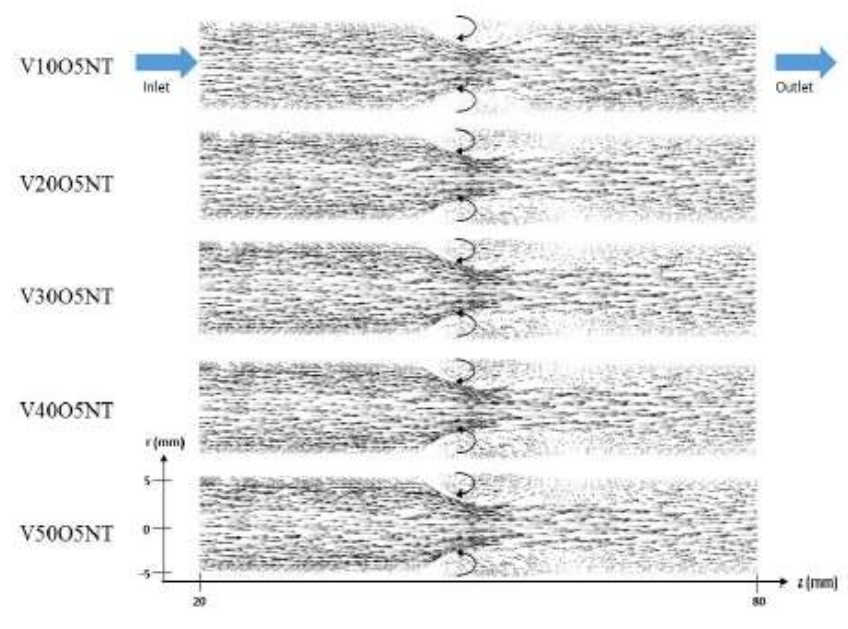

Fig. 7. Velocity Vector for each different Value of Inlet Velocity.

Fig. 7 shows the velocity vector for five cases with $50 \%$ of valve orifice along with the different inlet velocity. Based on the figure, the arrow means the flow of the stream. At the back of the valve, there are arrows which flow opposite to the zdirection which proves the back flow of blood at the cusps of the vein. The number of vector change slightly with the increasing value of inlet velocity. Here, V10O5NT shows uniform vector region along the stream. While, there are low vector region of backflow of vector at the cusps area. As velocity increasing, the vector region become stronger and the region become larger as shown by V50O5NT. The number of vector also decrease as it passing through the valve. In addition, the reversed flow region grows and expand with the increasing value of velocity. In case the velocity value is low, the condition could lead to the thrombosis development.

Vorticity contour of five cases as shown in Fig. 8 was simulated with $50 \%$ valve orifice and has different value of inlet velocity. The blue color indicates the blood particles which in the clockwise rotation, while red color indicates the anticlockwise rotation. The scaled legend showing the highest frequency value of 150 per second while the lowest vorticity frequency of -150 per second. From the all cases, it can be visualized that the vorticity near wall is slowly build up with the increasing of inlet velocity. V10O5NT shows very small region of particles moving at the valves. Meanwhile, the particles movement in V50O5NT shows the highest value of frequency due to the increasing of inlet velocity. Other than that, due to the flow separation at the valve edges, vortices were forming behind the cusps. The separation flow later reattaches at the sinus wall following the center stream at the lumen. In conclusion, low inlet velocity giving zero frequency of vorticity which most of the particles concentrating on the cusps area of vessel wall.

\section{B. Effect of Different Valve Orifice with $1 \mathrm{~mm}$ Thrombus}

The existence of thrombus able to completely or partially block the movement of blood particles. This section of result aiming to portray the effect of valve orifice on the thrombus formation of $1 \mathrm{~mm}$ in size. 


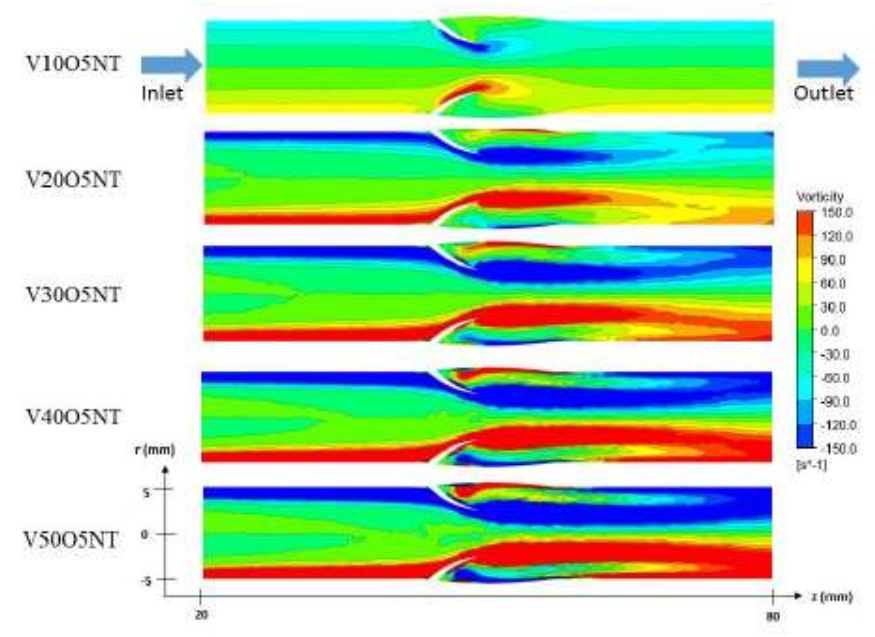

Fig. 8. Vorticity Contour Plot for five different Velocities.

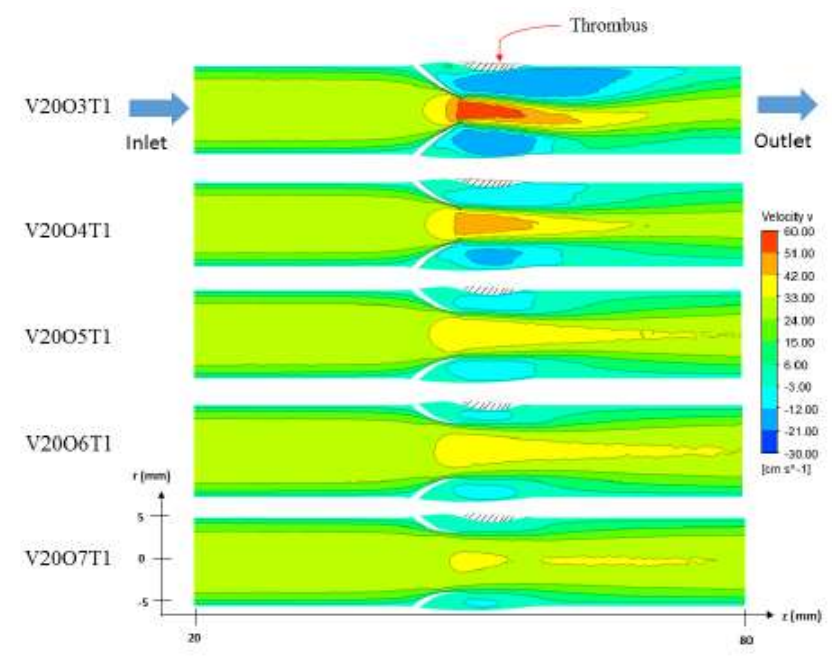

Fig. 9. Velocity Contour Plot for Five Cases with $1 \mathrm{~mm}$ Thrombus.

Fig. 9 demonstrate the velocity contour plot for five different valve orifice with the presence of thrombus. Each cases carried same inlet velocity which is $20 \mathrm{~cm} / \mathrm{s}$. Here, velocity value spotted to be increasing at the stenosis area. However, for case V20O3T1, the blood distribution slightly different from the normal flow. V20O3T1 shows high negative velocity region at the thrombus location. This prove that more particles are moving in opposite direction of the normal flow. Furthermore, the degree of reverse flow becomes worse with the decreasing of the valve orifice size. This indicates that the thrombus region in stenosis area causing more complex fluid flow.

Fig. 10 shows the velocity profile for each cases with different size of valve orifice. V20O3T1 showing fully developed velocity profile at the section A and B. While section $\mathrm{C}$ has developed negative or reverse flow zone as the fluid passing through valve area due to the sudden orifice of the vessel. Section D shows asymmetric velocity profile because of obstruction by the thrombus. The distorted velocity profile continues until section $\mathrm{E}$. The same pattern at the section A and B occurs in V20O4T1, V20O5T1, V20O6T1 and V20O7T1 which showing fully develop velocity profile. At the section $\mathrm{C}$ and $\mathrm{D}$, since the fluid just passing through the valve area, the boundary layer separation occurs where it can be observed that the fluid flow in unstable velocity value. However, the reverse flow on negative velocity value is changing with the increment of valve orifice.

Based on the Fig. 11, the positive axial velocity occupied the lumen of vessel. While the negative velocity appeared at the cusps area. The blood flowed backward as the blood occupied the cusps. V20O3T1 shows stronger velocity vector at the thrombus area since it has smaller size of orifice. Other than that, the vector size at the cusps area also decreasing with the increasing of the valve orifice. The number of vector also decrease as it passing through the valve. Here, it can be seen the reversed flow region grows and expend with the decreasing value of valve orifice.

The results in Fig. 12 show uniform pattern of vorticity contour near wall. More particles can be spotted reside at the wall area and at the edge of valve leaflets. Due to the narrowing of a vessel, high vorticity region was formed at the valve area. V20O3T1 shows slightly different pattern of vorticity as the blood passing through the thrombus area. Since it has smaller size of the orifice, the size of the vorticity region become wider compared to the other cases. It could be concluded that the vorticity region become smaller as the valve orifice become wider.

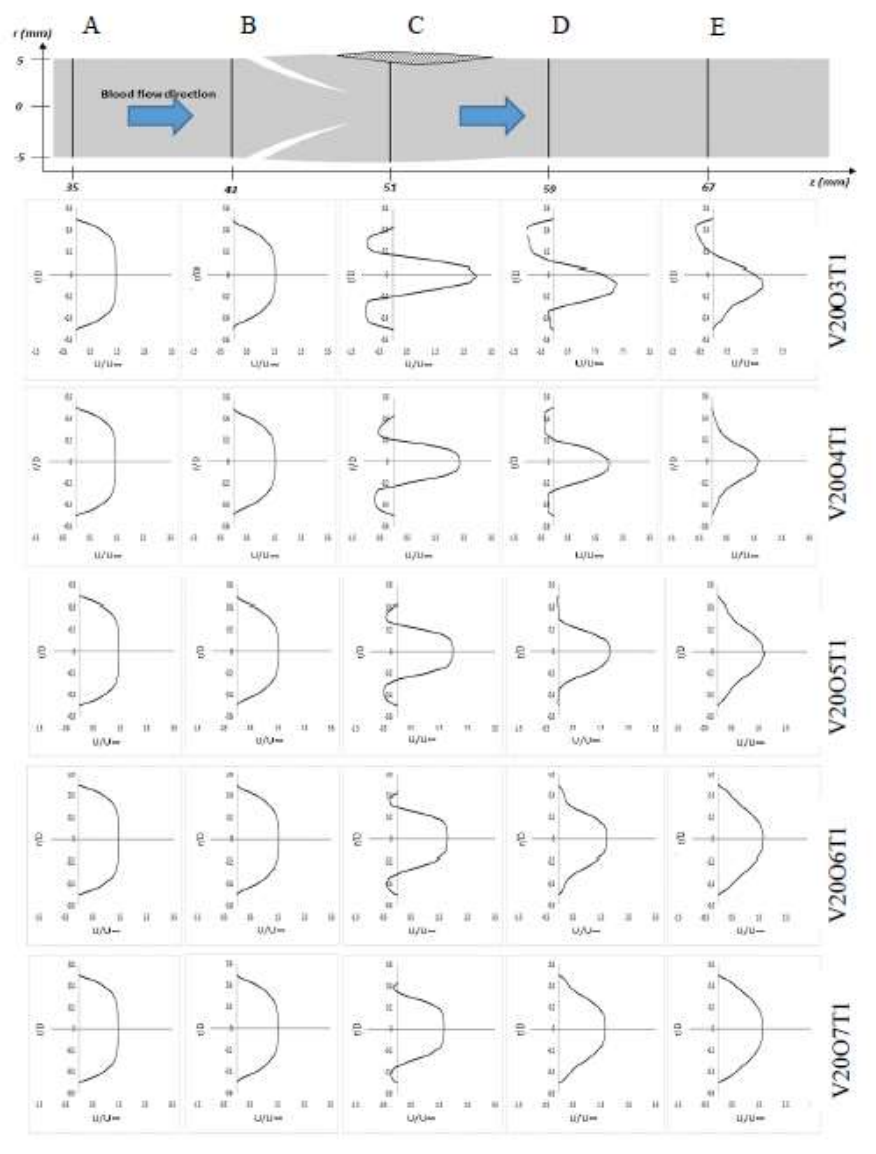

Fig. 10. Velocity Profile with $1 \mathrm{~mm}$ Thrombus. 


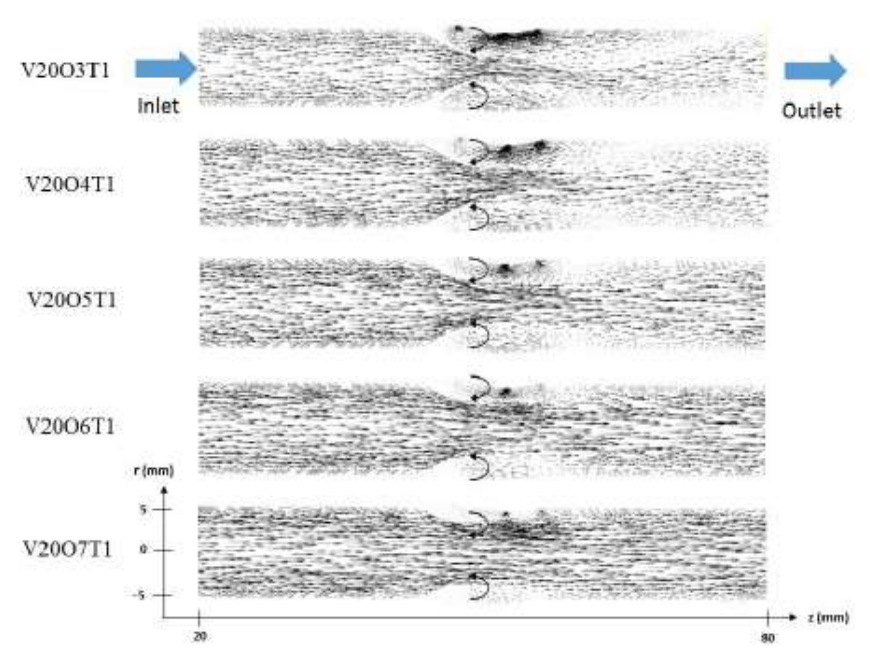

Fig. 11. Velocity Vector for Five different Valve Orifice with the Presence of $1 \mathrm{~mm}$ Thrombus Size.

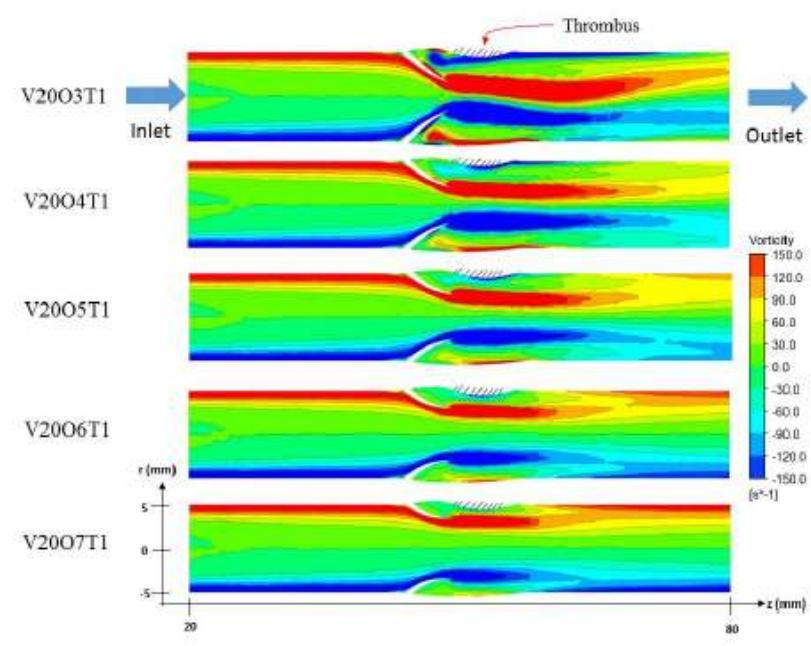

Fig. 12. Vorticity Contour Plot for Five different Valve Orifice with the Presence of $1 \mathrm{~mm}$ of Thrombus Size.

\section{Effect of Different Valve Orifice with $3 \mathrm{~mm}$ Thrombus}

This section was simulated to observe the effect of valve opening with the existence of $3 \mathrm{~mm}$ thrombus to the velocity of blood in the vein, which each cases carried the same inlet velocity of $20 \mathrm{~cm} / \mathrm{s}$.

From Fig. 13, an obvious pattern can be observed where the high velocity region only can be seen from the vessel with the smallest size of valve opening which is from V20O3T3. The high velocity region can be seen losing its momentum as the valve opening size increase. This could be concluded that blood flowing before the valve region is flow in a fully developed velocity profile. When the blood pass through the valve orifice, the velocity increase as it has to pass through the smaller area. The flow can be seen still accelerating as it passing through the thrombus area. Therefore, this shows that the increment of valve opening resulted on the decrement of the blood flow velocity in the vein. It also shows the unstable flow velocity at the thrombus area due to the disturbed flow velocity.

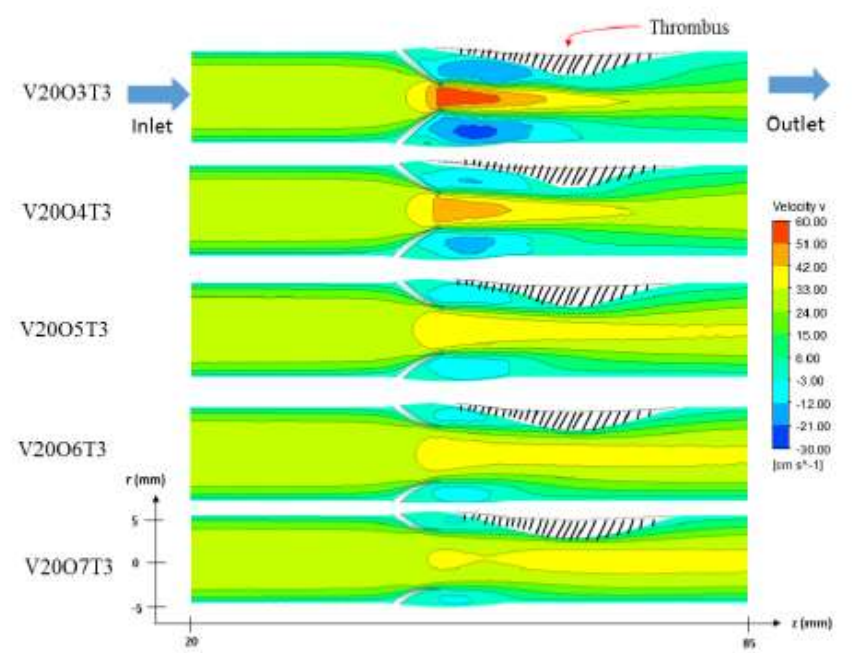

Fig. 13. Velocity Contour Plot for Five Cases with $3 \mathrm{~mm}$ Thrombus.

From the velocity profile shown in Fig. 14, each cases show the same pattern at section A and B with fully developed velocity profile. While section $\mathrm{C}$ and $\mathrm{D}$ has produced negative or reverse flow zone in V20O3T3 and V20O4T3 due to the obstruction by the thrombus. However, the reverse flow in section D shows decrement in the increment of valve orifice. In addition, the unstable of velocity profile can be spotted as the fluid is further away from the valve.

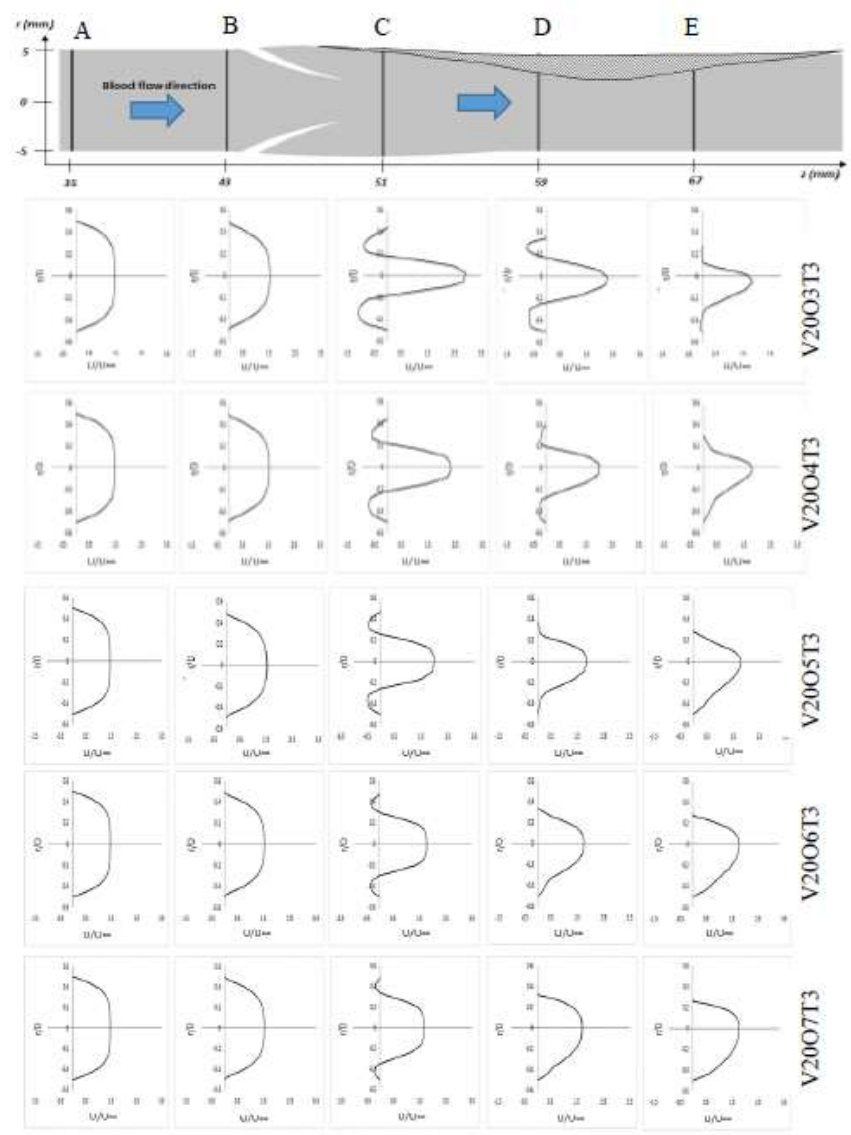

Fig. 14. Velocity Profile with $3 \mathrm{~mm}$ Thrombus. 


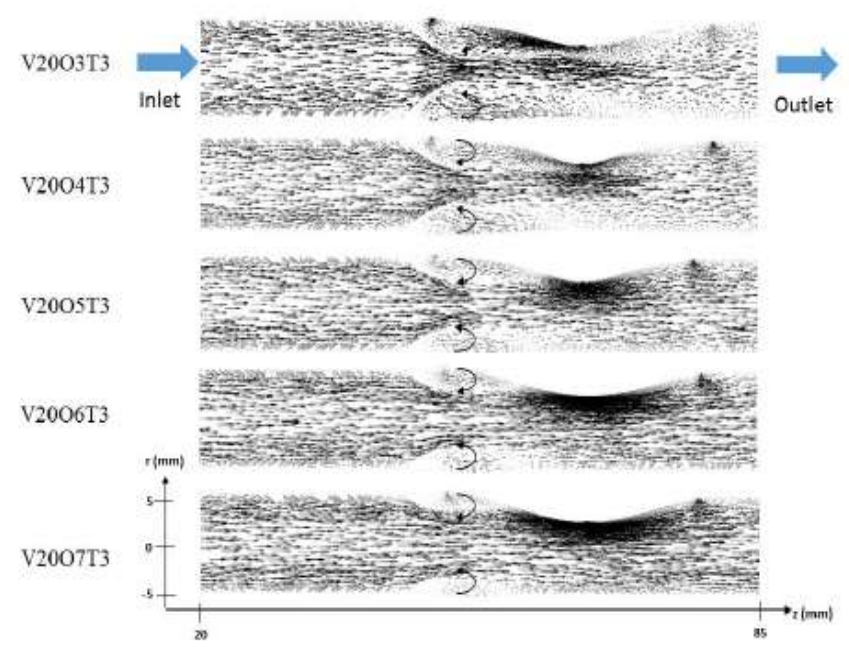

Fig. 15. Velocity Vector for five different Valve Orifice with the Presence of 3 mm Thrombus Size.

Fig. 15 shows the velocity vector for five cases with different size of valve orifice with the presence of $3 \mathrm{~mm}$ thrombus. The number of vector change slightly with the increasing of valve orifice. As the size of valve orifice increasing, the vortices become stronger at the thrombus area which can be seen from V20O7T3. The number of vector also decrease as it passing through the valve. Furthermore, the reversed flow region grows and expand with the decreasing value of orifice.

Fig. 16 demonstrates the vorticity contour plot of the five cases. It is clearly shows that the valve orifice size increased disproportionally to the frequency of vorticity. V20O7T3 shows very small region of particles moving at the valves area compared to the V20O3T3 where more particles can be spotted reside at the wall area and at the edge of valve leaflets.

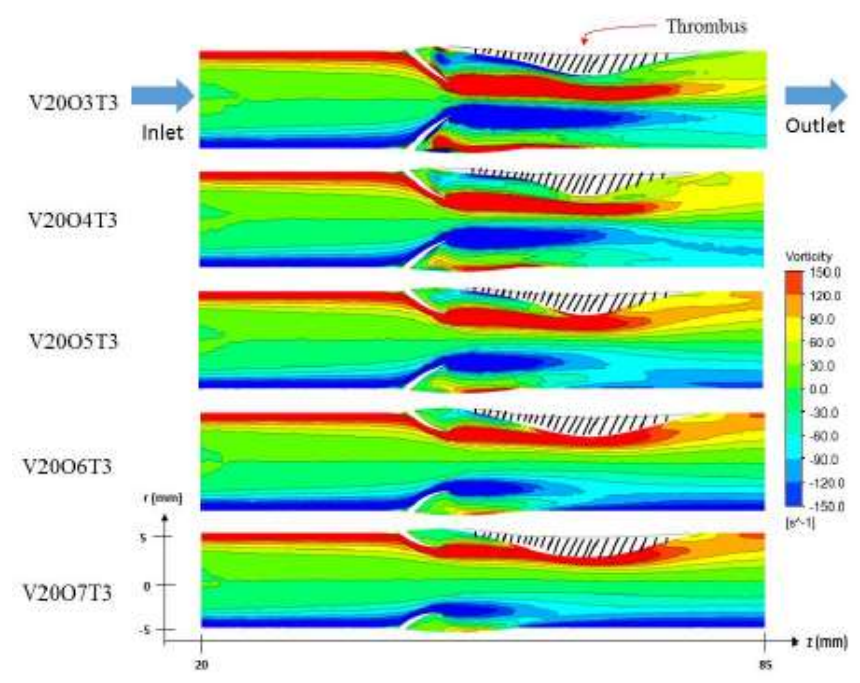

Fig. 16. Vorticity Contour Plot for Five different Valve Orifice with the Presence of $3 \mathrm{~mm}$ of Thrombus Size.

\section{DISCUSSIONS}

The endothelial damage is one of the risk factor of the thrombus formation. Previous work in [1] has agreed where the vascular wall contribute to the propagation of thrombus size. Moreover, the existence of thrombus in the vessel is also assumed to be the factor of vessel damage. Formation of thrombus has been discussed as a results from clot or plaque rupture which then leading to platelet aggregation [25]. All those risk factors are consolidating along with blood flow changes in blood vessels that later affect the serious brain damage. Classically, other risk factors that implicated in thrombosis are hypertension, high cholesterol, and smoking which slightly associated with an increased risk of stroke. At the end, this study tries to predict the feasibility of some of the risk factors to the thrombus formation.

Here, it can be concluded that in all cases, blood flowing prior to the valve region flowed in a fully developed velocity profiles. When the blood pass through the valve orifice, the velocity increases as it has to pass through the smaller area. The flow can be seen still accelerating as it passing through the thrombus area and increasing proportionally to the thrombus size. Other than that, the disturbed flow velocity can be seen at the thrombus area regardless the inlet velocity value. In addition, for the velocity profile $30 \%$ and $40 \%$ of valve orifice, it shows the unstable condition of velocity due to the opening size that might be considered as narrow space for the flow to pass through. To be concluded, the existence of thrombus inside the stenotic vessel causing more complex flow and may lead to the growth of thrombus.

\section{CONCLUSION}

The study was conducted to visualize the mechanism of blood in vein specifically popliteal vein. The computational fluid dynamics (CFD) modelling has been used to evaluate specific parameter which can affect the blood performance such as the sizes of valve orifice, inlet velocity and the presence of thrombus in the vein. In respect of CFD, the haemodynamics parameters such as velocity profile, velocity contour, velocity vector, vorticity contour was used to clearly visualize changes in blood flow as it follows the real condition of blood. The evaluation session on the vein was conducted with the presence of thrombus in two sizes that obviously obstruct the blood flow which then contribute to the thrombus alteration.

In future, it is recommended to simulate the case study using pulsatile blood flow instead of a steady blood flow. Since the wall of the vessel is assumed as rigid, it is a fact that vessel wall of a vein is collapsible. Thus, it is also suggested to study the effects of vessel wall to the blood flow. In the nutshell, present study has clearly showed the effect of blood flow velocity on thrombus development in the vessel.

\section{ACKNOWLEDGMENT}

Authors would like to thank the Ministry of Higher Education Malaysia for supporting this research under Fundamental Research Grant Scheme Vot. No. FRGS/1/2018/TK04/UTHM/02/24. 


\section{REFERENCES}

[1] T. Esmon, "Basic mechanisms and pathogenesis of venous thrombosis." Blood Reviews, vol. 23, pp. 225-229, 2009.

[2] D. R. Kumar, E. R. Hanlin, I. Glurich, J. J. Mazza, and S. H. Yale, "Virchow's contribution to the understanding of thrombosis and cellular biology," Clin. Med. And Research, vol. 8, pp. 168-172, 2010.

[3] P. C. Bennett, S. H. Silverman, P. S. Gill, and G.Y.H, "Peripheral arterial disease and Virchow's triad," Thrombosis and Haemostasis, vol.101, pp. 1032-1040, 2009.

[4] S. Khaladkar, D. Thakkar, K. Shinde, D. Thakkar, H. Shrotri, and V. Kulkarni, "Deep vein thrombosis of the lower limbs: A retrospective analysis of doppler ultrasound findings," Med. J. of Dr Dr. D.Y. Patil University, vol. 7, pp. 612-619, 2014.

[5] B. K. Zierler, "Ultrasonography and diagnosis of venous thromboembolism," Circulation, vol. 109, pp. 1-14, 2004.

[6] R. Hajar, "Risk factors for coronary artery disease: Historical perspectives," Heart Views, vol. 18, pp. 109-114, 2017.

[7] N. Ibrahim, W. N. Wan Zakaria, N. Aziz, and M. K. Abdullah, "Construction of phantom mimic vessel for study of human vessel conditions in deep vein thrombosis," IFMBE Proceedings, vol. 46, pp. 402-404, 2015.

[8] M. Selmi, H. Belmabrouk, and A. Bajahzar, "Numerical study of the blood flow in a deformable human aorta," Appl. Sci., vol. 9, pp. 12161227, 2019.

[9] N. Aziz, N. Ibrahim, M. K. Abdullah, N. H. I Mat Harun, "Computational fluid dynamics simulation on blood velocity and vorticity of venous valve behaviour,". Lecture Notes in Electrical Engineering, vol. 398, pp. 617-625, 2017.

[10] Lili Niu, Ming Qian, Wei Yang, Long Meng, Yang Xiao, Kelvin K.L. Wong, Derek Abbott, Xin Liu, Hairong Zheng, "Surface roughness detection of arteries via texture analysis of ultrasound images for early diagnosis of atherosclerosis," PLOS ONE, vol. 8, pp. 1-11, 2013.

[11] Angels Betriu-Bars, Elvira Fernandez-Giraldez, "Carotid ultrasound for the early diagnosis of atherosclerosis in chronic kidney disease," Nefrologia, vol. 32, pp. 7-11, 2012.

[12] Md. Rejaul Haque, Md. Emran Hossain, A.B.M. Toufique Hasan, "Effect of non-Newtonian behaviour on fluid structural interaction for flow through a model stenosed artery," Procedia Engineering, vol. 90, pp. 358-363, 2014.

[13] Z. Tyfa, D. Obidowski, P. Reorowicz, L. Stefanczyl, J. Fortuniak, K. Jozwik, "Numerical simulations of the pulsatile blood flow in the different types of arterial fenestrations: comparable anaylsis of multiple vascular geometries," Biocybernetics and Biomedical Engineering, vol. 38, pp. 228-242, 2018.

[14] N. El Khatib, O. Kafi, A. Sequeira, S. Simakov, Yu. Vassilevski, V. Volpert, "Mathematical modelling of atherosclerosis," Math. Model Nat. Phenom, vol. 14, pp. 1-25, 2019.

[15] R. Morris and J. P. Woodcock, "Evidence-based compression. Prevention of stasis and deep vein thrombosis," Ann. Surg., vol. 239, pp. 162-171, 2004.

[16] Y. J. Zhang, T. Struffert, J. Hornegger, “ Simulation of the interaction between blood flow and atherosclerosis plaque," IEEE Eng. Med. Biol. Soc., pp. 1699-1702, August 2007 [29thAnnual Conf. IEEE EMBS Cite International, p. 1699, 2007].

[17] K. Chandran, S. Rittgers, A. Yoganathan, Biofluid Mechanis. Boca Raton, CRC/Taylor\&Francis, 2007.

[18] Baskurt O.K., "Pathophysiological significance of blood rheology," Turkish Journal of Medical Sciences, vol. 33, pp. 347-355, 2003.

[19] Y. Jiang, J. Zhang, W. Zhao, "Effect of the inlet conditions and blood models on accurate prediction of hemodynamics in the stented coronary arteries," AIP Advances, vol. 5, pp. 057109-1-057109-9, 2015.

[20] Vinoth R., Kumar D., Raviraj A., Vijay Shankar CS, "Non-newtonian and newtonian blood flow in human aorta," Biomedical Research, vol. 28, pp. 3194-3203, 2017.

[21] K. D. Dennis, D. F. Kallmes, and D. Dragomir-daescu, "Cerebral aneurysm blood flow simulations are sensitive to basic solver settings," J. Biomech., vol. 57, pp. 46-53, 2017.

[22] L. S. Hong, M. A. Hisham Mohd Adib, M. Uzair Matalif, M. Shafie Abdullah, N. Hartini Mohd Taib, R. Hassan, "Modelling and simulation of blood flow analysis on simplified aneurysm models," IOP Conf. Series: Materials Science and Engineering, vol. 917, pp. 1-10, 2020.

[23] Yue Zhou, Chunhian Lee, Jingying Wang, "The computational fluid dynamics analyses on hemodynamic characteristics in stenosed arterial model," J. Health Eng., vol. 2018, pp. 1-6, 2018.

[24] Ana Paul N., Fernando Silva M., Frederic P., Alberto M., Ignacio L., Jean-Marc G., Cecile p., Charles A. S., Ayache B., "A clinically aligned experimental approach for quantitative characterization of patientspecific cardiovascular model," AIP Advances, vol. 10, pp. 045106-1045196-10, 2020.

[25] M. Koupenova, B. E. Kehrel, H. A. Corkrey, J. E. Freedman, "Thrombosis and platelets: an update," European Heart Journal, vol. 38, pp. 785-791, 2017. 